\title{
A PRINCIPAL STRESS CONTOUR FOR NONLINEAR ELASTIC DEFORMATIONS
}

\author{
BY \\ JINGYU SHI \\ Nottingham University, United Kingdom and Queensland University, Australia
}

\begin{abstract}
We seek special principal stress contours, for a nonlinear elastic plane deformation, on which both the principal stresses are constant and the principal axes do not change directions. It is found that they must be straight lines and that the lines must be parallel to one another or intersect at one point. It is also shown that the principal stress contours in the deformation for the straightening of a circular arc are such straight lines.
\end{abstract}

1. Introduction. The principal stress and strain trajectories for a nonlinear elastic plane deformation have been discussed by Ogden [1]. He obtained a system of four first-order partial differential equations governing the principal stretches of the plane deformation and the two angles that define the orientation of the Lagrangean and Eulerian principal axes of the deformation.

Assume in a nonlinear plane deformation of isotropic elastic material, the two in-plane principal stresses (or strains) and the angle representing the direction of the Eulerian principal axes can be expressed as functions of a single parameter, which in turn is a function of the position coordinates of the points. Letting the parameter be equal to some constant, we can obtain a curve in the plane of the deformation, and then along the curve the two principal stresses (and strains) are constant and the Eulerian principal axes do not change direction. Thus the curve is a special principal stress contour. Different values of the parameter produce different contours. In this paper we will discuss the properties of the contours.

To do so, we first in Sec. 2 express the deformation gradient in terms of principal stretches and the angles that define the orientations of the Lagrangean and Eulerian principal axes of the deformation. Then we write the constitutive equations for compressible isotropic elastic materials, the equilibrium equations, and compatibility equations. In Sec. 3 we will search for the special principal stress contours, in the deformed configuration, on which the principal stresses (and strains) are constant and the Eulerian axes do not change direction. We find that the contours must be straight lines and that the lines must be parallel to one another or intersect at one point. At the same time, we establish the governing equations in terms of the parameter for the 
deformation that has this property. Finally in Sec. 4 we show that the straightening of a circular arc is an inhomogeneous deformation that has these principal stress contours. The principal stress contours lie along the straight lines deformed from the circumferential curves. This result is true for any isotropic material, although the precise expression for the deformation depends on the material properties.

2. Governing equations for nonlinear elastic deformations. We consider a cylinder of homogeneous, isotropically elastic material and establish a Cartesian coordinate system $\left(X_{1}, X_{2}, X_{3}\right)$ such that the $X_{3}$-axis is parallel to the generators of the cylinder and the $X_{1}, X_{2}$-axes lie in the plane perpendicular to the generators. We assume that the applied forces are such that the cylinder undergoes a deformation

$$
x_{1}=x_{1}\left(X_{1}, X_{2}\right), \quad x_{2}=x_{2}\left(X_{1}, X_{2}\right), \quad x_{3}=\lambda X_{3},
$$

where $X_{i}$ and $x_{i}(i=1,2,3)$ are the initial and final coordinates of the same point respectively and $\lambda$ is constant. Then the $X_{3}$-axis is one of the principal axes of the deformation, and the corresponding principal stretch is $\lambda$. The remaining two principal axes lie in the $\left(X_{1}, X_{2}\right)$-plane. These latter axes and the corresponding principal stretches can be completely determined from the in-plane deformation gradient tensor [2]

$$
\mathbf{A}=\left(\begin{array}{ll}
\frac{\partial x_{1}}{\partial X_{1}} & \frac{\partial x_{1}}{\partial X_{2}} \\
\frac{\partial x_{2}}{\partial X_{1}} & \frac{\partial x_{2}}{\partial X_{2}}
\end{array}\right)
$$

It can be shown $[1,3,4]$ that this tensor can be expressed as

$$
\mathbf{A}=\left(\begin{array}{ll}
\lambda_{1} \cos \varphi \cos \psi+\lambda_{2} \sin \varphi \sin \psi & \lambda_{1} \sin \varphi \cos \psi-\lambda_{2} \cos \varphi \sin \psi \\
\lambda_{1} \cos \varphi \sin \psi-\lambda_{2} \sin \varphi \cos \psi & \lambda_{1} \sin \varphi \sin \psi+\lambda_{2} \cos \varphi \cos \psi
\end{array}\right) .
$$

Here $\lambda_{1}$ and $\lambda_{2}$ are the principal stretches in the $\left(X_{1}, X_{2}\right)$-plane, and $\varphi$ and $\psi$ describe the orientations of the Lagrangean and Eulerian principal axes in the plane. Then the inverse of $\mathbf{A}$ has the form

$$
\mathbf{A}^{-1}=\left(\begin{array}{ll}
\lambda_{1}^{-1} \cos \varphi \cos \psi+\lambda_{2}^{-1} \sin \varphi \sin \psi & \lambda_{1}^{-1} \cos \varphi \sin \psi-\lambda_{2}^{-1} \sin \varphi \cos \psi \\
\lambda_{1}^{-1} \sin \varphi \cos \psi-\lambda_{2}^{-1} \cos \varphi \sin \psi & \lambda_{1}^{-1} \sin \varphi \sin \psi+\lambda_{2}^{-1} \cos \varphi \cos \psi
\end{array}\right) .
$$

Since the material is isotropic, the principal axes of the stress coincide with those of the strain. The Piola-Kirchhoff (or nominal) stress tensor in the plane has the form

$$
\mathbf{S}=\left(\begin{array}{ll}
S_{11} & S_{12} \\
S_{21} & S_{22}
\end{array}\right)=\left(\begin{array}{ll}
s_{1} \cos \varphi \cos \psi+s_{2} \sin \varphi \sin \psi & s_{1} \sin \varphi \cos \psi-s_{2} \cos \varphi \sin \psi \\
s_{1} \cos \varphi \sin \psi-s_{2} \sin \varphi \cos \psi & s_{1} \sin \varphi \sin \psi+s_{2} \cos \varphi \cos \psi
\end{array}\right),
$$

where $s_{1}$ and $s_{2}$ are the Piola-Kirchhoff (or nominal) principal stresses in the plane of the deformation. From the relation between the Cauchy stress tensor $\mathbf{T}$ and the Piola-Kirchhoff stress tensor $\mathbf{S}$, i.e.,

$$
\mathbf{T}=(\operatorname{det} \mathbf{A})^{-1} \mathbf{A S}
$$


(see Spencer [5]), the Cauchy stress tensor has the form

$$
\mathbf{T}=\left(\begin{array}{ll}
t_{11} & t_{12} \\
t_{21} & t_{22}
\end{array}\right)=\left(\begin{array}{ll}
t_{1} \cos ^{2} \psi+t_{2} \sin ^{2} \psi & \left(t_{1}-t_{2}\right) \cos \psi \sin \psi \\
\left(t_{1}-t_{2}\right) \cos \psi \sin \psi & t_{1} \sin ^{2} \psi+t_{2} \cos ^{2} \psi
\end{array}\right),
$$

in which $t_{1}$ and $t_{2}$ are the Cauchy principal stresses in the plane of the deformation.

The stress-strain relations for an isotropically elastic compressible material with the plane deformation are (see Spencer [5])

$$
\begin{gathered}
t_{\alpha \beta}=a_{0} \delta_{\alpha \beta}+a_{1} B_{\alpha \beta}+a_{2} B_{\alpha \gamma} B_{\gamma \beta}, \\
t_{3 \alpha}=t_{\alpha 3}=0 \quad(\alpha, \beta=1,2), \\
t_{33}=a_{0}+a_{1} \lambda^{2}+a_{2} \lambda^{4},
\end{gathered}
$$

where

$$
\begin{gathered}
B_{\alpha \beta}=\frac{\partial x_{\alpha}}{\partial X_{\gamma}} \frac{\partial x_{\beta}}{\partial X_{\gamma}}, \\
\left\{\begin{array}{l}
a_{0}=2 I_{3}^{1 / 2} \partial W / \partial I_{3}, \\
a_{1}=2 I_{3}^{-1 / 2}\left(\partial W / \partial I_{1}+I_{1} \partial W / \partial I_{2}\right), \\
a_{2}=-2 I_{3}^{-1 / 2} \partial W / \partial I_{2},
\end{array}\right. \\
\left\{\begin{array}{l}
I_{1}=B_{\alpha \alpha}+\lambda^{2}, \\
I_{2}=\left[\left(B_{\alpha \alpha}+\lambda^{2}\right)^{2}-\left(B_{11}^{2}+B_{22}^{2}+2 B_{12}^{2}+\lambda^{4}\right)\right] / 2, \\
I_{3}=\lambda^{2}\left(B_{11} B_{22}-B_{12}^{2}\right),
\end{array}\right.
\end{gathered}
$$

and $W=W\left(I_{1}, I_{2}, I_{3}\right)$ is the strain-energy function for the material.

If body forces are absent, then the equilibrium equations in the deformed configuration are

$$
\frac{\partial t_{11}}{\partial x_{1}}+\frac{\partial t_{12}}{\partial x_{2}}=0, \quad \frac{\partial t_{21}}{\partial x_{1}}+\frac{\partial t_{22}}{\partial x_{2}}=0 .
$$

For the continuity of the deformation field $x_{1}, x_{2}$, the stress and strain must satisfy certain compatibility conditions. These conditions are obtained by substituting (2.3) into

or $(2.4)$ into

$$
\frac{\partial^{2} x_{1}}{\partial X_{1} \partial X_{2}}=\frac{\partial^{2} x_{1}}{\partial X_{2} \partial X_{1}}, \quad \frac{\partial^{2} x_{2}}{\partial X_{1} \partial X_{2}}=\frac{\partial^{2} x_{2}}{\partial X_{2} \partial X_{1}},
$$

$$
\frac{\partial^{2} X_{1}}{\partial x_{1} \partial x_{2}}=\frac{\partial^{2} X_{1}}{\partial x_{2} \partial x_{1}}, \quad \frac{\partial^{2} X_{2}}{\partial x_{1} \partial x_{2}}=\frac{\partial^{2} X_{2}}{\partial x_{2} \partial x_{1}} \text {. }
$$

3. Special principal stress contours and governing equations for associated deformations. If we assume that in a plane deformation, $t_{1}, t_{2}$, and $\psi$ can be expressed as continuous functions:

$$
t_{1}=t_{1}(\alpha), \quad t_{2}=t_{2}(\alpha), \quad \psi=\psi(\alpha)
$$

of a parameter $\alpha$, which in turn is a continuous function of $x_{1}$ and $x_{2}$ :

$$
\alpha=\alpha\left(x_{1}, x_{2}\right),
$$


then along the curve $\alpha\left(x_{1}, x_{2}\right)=$ constant, in the deformed configuration, the principal stresses $t_{1}$ and $t_{2}$ are constant and the Eulerian principal axes do not change direction. The question is whether or not there exists such a deformation and what is the shape of the curve. It is well known that, in the case of a pure homogeneous deformation, the principal stresses are constant and the principal axes do not change direction throughout the plane of the deformation; therefore the expressions (3.1) and (3.2) are valid for any $\alpha$. In the following we will show that if there is such an inhomogeneous deformation, then the curve must be a straight line. Giving different values to $\alpha$ will yield different straight lines, so we have a family of straight lines. It will further be shown that the lines must be parallel or all intersect at a single point. Then in the next section, we will give an example of such an inhomogeneous deformation.

Substituting (2.7) with (3.1) into the equilibrium equations (2.11), we have

$$
\begin{aligned}
& \frac{d}{d \alpha}\left[t_{1} \cos ^{2} \psi+t_{2} \sin ^{2} \psi\right] \frac{\partial \alpha}{\partial x_{1}}+\frac{d}{d \alpha}\left[\left(t_{1}-t_{2}\right) \cos \psi \sin \psi\right] \frac{\partial \alpha}{\partial x_{2}}=0, \\
& \frac{d}{d \alpha}\left[\left(t_{1}-t_{2}\right) \cos \psi \sin \psi\right] \frac{\partial \alpha}{\partial x_{1}}+\frac{d}{d \alpha}\left[t_{1} \sin ^{2} \psi+t_{2} \cos ^{2} \psi\right] \frac{\partial \alpha}{\partial x_{2}}=0 .
\end{aligned}
$$

If there is a nonconstant continuous function $\alpha=\alpha\left(x_{1}, x_{2}\right)$, i.e., $\partial \alpha / \partial x_{1} \neq 0$ and/or $\partial \alpha / \partial x_{2} \neq 0$, then the determinant of the coefficients of $\partial \alpha / \partial x_{1}$ and $\partial \alpha / \partial x_{2}$ must vanish; i.e.,

$\frac{d}{d \alpha}\left[t_{1} \cos ^{2} \psi+t_{2} \sin ^{2} \psi\right] \frac{d}{d \alpha}\left[t_{1} \sin ^{2} \psi+t_{2} \cos ^{2} \psi\right]-\left\{\frac{d}{d \alpha}\left[\left(t_{1}-t_{2}\right) \cos \psi \sin \psi\right]\right\}^{2}=0$, which yields, after some manipulation,

$$
t_{1}^{\prime} t_{2}^{\prime}-\left(t_{1}-t_{2}\right) \psi^{\prime}=0
$$

Here the prime denotes the derivatives with respect to $\alpha$. This is one governing equation for the state of stress in the inhomogeneous deformation in terms of $\alpha$. When (3.4) is satisfied, we can solve from (3.3) for $\partial \alpha / \partial x_{1}$ and $\partial \alpha / \partial x_{2}$. The solutions are

$$
\begin{aligned}
& \frac{\partial \alpha}{\partial x_{1}}=h\left(x_{1}, x_{2}\right) f_{1}(\alpha), \\
& \frac{\partial \alpha}{\partial x_{2}}=-h\left(x_{1}, x_{2}\right) f_{2}(\alpha),
\end{aligned}
$$

where $h\left(x_{1}, x_{2}\right)$ is an arbitrary function of $x_{1}$ and $x_{2}$, and $f_{1}(\alpha)$ and $f_{2}(\alpha)$ are defined as

$$
\begin{aligned}
& f_{1}(\alpha)=\frac{d}{d \alpha}\left[\left(t_{1}-t_{2}\right) \cos \psi \sin \psi\right], \\
& f_{2}(\alpha)=\frac{d}{d \alpha}\left[t_{1} \cos ^{2} \psi+t_{2} \sin ^{2} \psi\right] .
\end{aligned}
$$

If a value for $\alpha$ is specified, say $C$, then $\alpha\left(x_{1}, x_{2}\right)=C$ represents a curve in the $\left(x_{1}, x_{2}\right)$-plane, and the slope of its tangent is given by

$$
\frac{d x_{2}}{d x_{1}}=-\frac{\partial \alpha / \partial x_{1}}{\partial \alpha / \partial x_{2}}
$$


From (3.5) we can see that the slope of the curve we want is

$$
\frac{d x_{2}}{d x_{1}}=\frac{f_{1}(C)}{f_{2}(C)} \equiv M(C)
$$

Along the curve, $C$ is a constant, and hence the slope $M(C)$ at any point of the curve is constant too. This implies that the curve must be a straight line. The straight line may be expressed as

$$
x_{2}=x_{1} M(C)+D(C),
$$

where $D(C)$ is a constant, representing the position of the intersecting point of the straight line and the $x_{2}$-axis. Different constants $C$ correspond to different lines; different lines may have different slopes and different intersecting points with the coordinate axis.

The constant $C$ is the value chosen for $\alpha$; thus Eq. (3.9) can be written in terms of $\alpha$ :

$$
x_{2}=x_{1} M(\alpha)+D(\alpha) \text {. }
$$

We can see from (3.6) and (3.8) that the slope $M(\alpha)$ of the straight line is related to the state of stress by

$$
\frac{d}{d \alpha}\left[\left(t_{1}-t_{2}\right) \cos \psi \sin \psi\right]=M(\alpha) \frac{d}{d \alpha}\left[t_{1} \cos ^{2} \psi+t_{2} \sin ^{2} \psi\right],
$$

but $D(\alpha)$ is arbitrary. Actually, arbitrariness of $D(\alpha)$ is equivalent to that of $h\left(x_{1}, x_{2}\right)$. To see this, differentiating both sides of (3.10) with respect to $x_{1}$ and $x_{2}$ respectively, we find

$$
\begin{aligned}
\frac{\partial \alpha}{\partial x_{1}} & =-\frac{M(\alpha)}{D^{\prime}(\alpha)+x_{1} M^{\prime}(\alpha)}, \\
\frac{\partial \alpha}{\partial x_{2}} & =\frac{1}{D^{\prime}(\alpha)+x_{1} M^{\prime}(\alpha)} .
\end{aligned}
$$

Then comparing these with (3.5), we have

$$
h\left(x_{1}, x_{2}\right)=-\left[\left(D^{\prime}+x_{1} M^{\prime}\right) f_{2}\right]^{-1} .
$$

It can also be shown that

$$
\frac{\partial^{2} \alpha}{\partial x_{1} \partial x_{2}}=\frac{\partial^{2} \alpha}{\partial x_{2} \partial x_{1}}
$$

for any $D(\alpha)$.

We have, from the equilibrium consideration, two equations that relate five parameters $t_{1}, t_{2}, \psi, M$, and $D$. More equations can be obtained from the compatibility conditions. It follows, from the isotropy of the material, that the principal stretches can also be expressed as functions of $\alpha$ :

$$
\lambda_{1}=\lambda_{1}(\alpha), \quad \lambda_{2}=\lambda_{2}(\alpha) .
$$

Substituting (2.4) with (3.14) into (2.13), we have two equations from which we can solve for $\partial \varphi / \partial x_{1}$ and $\partial \varphi / \partial x_{2}$ in terms of $\lambda_{1}, \lambda_{2}, \psi, \alpha$, and their derivatives. 
These solutions are

$$
\begin{array}{r}
\frac{\partial \varphi}{\partial x_{1}}=-\lambda_{1} \lambda_{2}\left\{\left[-\frac{\cos \psi}{\lambda_{1}} \frac{d}{d \alpha}\left(\frac{\sin \psi}{\lambda_{1}}\right)+\frac{\sin \psi}{\lambda_{2}} \frac{d}{d \alpha}\left(\frac{\cos \psi}{\lambda_{2}}\right)\right] \frac{\partial \alpha}{\partial x_{1}}\right. \\
\left.+\left[\frac{d}{d \alpha}\left(\frac{\cos ^{2} \psi}{2 \lambda_{1}^{2}}\right)+\frac{d}{d \alpha}\left(\frac{\sin ^{2} \psi}{2 \lambda_{2}^{2}}\right)\right] \frac{\partial \alpha}{\partial x_{2}}\right\} \\
\frac{\partial \varphi}{\partial x_{2}}=-\lambda_{1} \lambda_{2}\left\{\left[-\frac{d}{d \alpha}\left(\frac{\sin ^{2} \psi}{2 \lambda_{1}^{2}}\right)-\frac{d}{d \alpha}\left(\frac{\cos ^{2} \psi}{2 \lambda_{2}^{2}}\right)\right] \frac{\partial \alpha}{\partial x_{1}}\right. \\
\left.+\left[\frac{\sin \psi}{\lambda_{1}} \frac{d}{d \alpha}\left(\frac{\cos \psi}{\lambda_{1}}\right)-\frac{\cos \psi}{\lambda_{2}} \frac{d}{d \alpha}\left(\frac{\sin \psi}{\lambda_{2}}\right)\right] \frac{\partial \alpha}{\partial x_{2}}\right\} .
\end{array}
$$

It follows from (3.12) that

$$
\frac{\partial \alpha}{\partial x_{1}}=-M(\alpha) \frac{\partial \alpha}{\partial x_{2}}
$$

Introducing (3.16) into (3.15), we have

$$
\frac{\partial \varphi}{\partial x_{1}}=g_{1}(\alpha) \frac{\partial \alpha}{\partial x_{2}}, \quad \frac{\partial \varphi}{\partial x_{2}}=g_{2}(\alpha) \frac{\partial \alpha}{\partial x_{2}},
$$

where

$$
\begin{array}{r}
g_{1}(\alpha)=\lambda_{1} \lambda_{2}\left\{\left[-\frac{\cos \psi}{\lambda_{1}} \frac{d}{d \alpha}\left(\frac{\sin \psi}{\lambda_{1}}\right)+\frac{\sin \psi}{\lambda_{2}} \frac{d}{d \alpha}\left(\frac{\cos \psi}{\lambda_{2}}\right)\right] M(\alpha)\right. \\
\left.-\left[\frac{d}{d \alpha}\left(\frac{\cos ^{2} \psi}{2 \lambda_{1}^{2}}\right)+\frac{d}{d \alpha}\left(\frac{\sin ^{2} \psi}{2 \lambda_{2}^{2}}\right)\right]\right\}, \\
g_{2}(\alpha)=\lambda_{1} \lambda_{2}\left\{\left[-\frac{d}{d \alpha}\left(\frac{\sin ^{2} \psi}{2 \lambda_{1}^{2}}\right)-\frac{d}{d \alpha}\left(\frac{\cos ^{2} \psi}{2 \lambda_{2}^{2}}\right)\right] M(\alpha)\right. \\
\left.-\left[\frac{\sin \psi}{\lambda_{1}} \frac{d}{d \alpha}\left(\frac{\cos \psi}{\lambda_{1}}\right)-\frac{\cos \psi}{\lambda_{2}} \frac{d}{d \alpha}\left(\frac{\sin \psi}{\lambda_{2}}\right)\right]\right\}
\end{array}
$$

are functions of $\alpha$. A continuous function $\varphi\left(x_{1}, x_{2}\right)$ can be obtained by integrating (3.17) when the following condition is satisfied:

$$
\frac{\partial}{\partial x_{2}}\left(g_{1}(\alpha) \frac{\partial \alpha}{\partial x_{2}}\right)=\frac{\partial}{\partial x_{1}}\left(g_{2}(\alpha) \frac{\partial \alpha}{\partial x_{2}}\right) .
$$

The condition (3.19) places a further restriction on the stresses. Expanding (3.19), we have

$$
g_{1}(\alpha) \frac{\partial^{2} \alpha}{\partial x_{2}^{2}}+g_{1}^{\prime}(\alpha)\left(\frac{\partial \alpha}{\partial x_{2}}\right)^{2}=g_{2}(\alpha) \frac{\partial^{2} \alpha}{\partial x_{1} x_{2}}+g_{2}^{\prime}(\alpha) \frac{\partial \alpha}{\partial x_{1}} \frac{\partial \alpha}{\partial x_{2}}
$$

and then making use of (3.13) and (3.16) yields

$$
\left[g_{1}(\alpha)+M(\alpha) g_{2}(\alpha)\right] \frac{\partial^{2} \alpha}{\partial x_{2}^{2}}+\left[g_{1}^{\prime}(\alpha)+M(\alpha) g_{2}^{\prime}(\alpha)+M^{\prime}(\alpha) g_{2}(\alpha)\right]\left(\frac{\partial \alpha}{\partial x_{2}}\right)^{2}=0,
$$


which can be rewritten as

$$
\frac{g_{1}^{\prime}+\left(M g_{2}\right)^{\prime}}{g_{1}+M g_{2}}=-\frac{\partial^{2} \alpha / \partial x_{2}^{2}}{\left(\partial \alpha / \partial x_{2}\right)^{2}}
$$

Introducing the second equation of (3.12) into this, we obtain

$$
\frac{g_{1}^{\prime}+\left(M g_{2}\right)^{\prime}}{g_{1}+M g_{2}}=\frac{D^{\prime \prime}(\alpha)+x_{1} M^{\prime \prime}(\alpha)}{D^{\prime}(\alpha)+x_{1} M^{\prime}(\alpha)} \text {. }
$$

Since the left-hand side is the function of $\alpha$ alone, so too is the right-hand side. Thus one of the following three must hold:

(1) $M^{\prime}(\alpha)=0$ while $D^{\prime}(\alpha) \neq 0$,

(2) $M^{\prime}(\alpha) \neq 0$ while $D^{\prime}=0$,

(3) $\quad M^{\prime}(\alpha) \neq 0, \quad D^{\prime}(\alpha) \neq 0 \quad$ while $\frac{D^{\prime \prime}(\alpha)}{D^{\prime}(\alpha)}=\frac{M^{\prime \prime}(\alpha)}{M^{\prime}(\alpha)}$.

The first one implies that the straight lines are parallel. The second one means that the straight lines intersect at a point on the $x_{2}$-axis. From the third condition, we have

$$
D(\alpha)=C_{0} M(\alpha)+C_{1} .
$$

Here $C_{0}$ and $C_{1}$ are constants. Introducing this into (3.10) we obtain

$$
x_{2}=M(\alpha)\left(x_{1}+C_{0}\right)+C_{1} .
$$

This implies that the lines intersect at one point $\left(-C_{0}, C_{1}\right)$ too, but not on the $x_{2}$ axis. So the straight lines must be parallel or intersect at one point. Accordingly the condition (3.20) becomes

$$
\begin{aligned}
& \frac{g_{1}^{\prime}+\left(M g_{2}\right)^{\prime}}{g_{1}+M g_{2}}=\frac{D^{\prime \prime}(\alpha)}{D^{\prime}(\alpha)} \quad \text { if } M^{\prime}(\alpha)=0, \\
& \frac{g_{1}^{\prime}+\left(M g_{2}\right)^{\prime}}{g_{1}+M g_{2}}=\frac{M^{\prime \prime}(\alpha)}{M^{\prime}(\alpha)} \quad \text { if } M^{\prime}(\alpha) \neq 0 .
\end{aligned}
$$

Integrating both sides with respect to $\alpha$, we have

$$
\begin{aligned}
& g_{1}(\alpha)+M(\alpha) g_{2}(\alpha)=C_{2} D^{\prime}(\alpha) \quad \text { if } M^{\prime}(\alpha)=0 \\
& g_{1}(\alpha)+M(\alpha) g_{2}(\alpha)=C_{3} M^{\prime}(\alpha) \text { if } M^{\prime}(\alpha) \neq 0
\end{aligned}
$$

Here $C_{2}$ and $C_{3}$ are arbitrary constants.

We note here that when $M^{\prime}(\alpha)=D^{\prime}(\alpha)=0$, all the straight lines collapse onto a single line since this is the only possibility if the lines are to be parallel $\left(M^{\prime}(\alpha)=0\right)$ and intersect at one point $\left(D^{\prime}(\alpha)=0\right)$. This singular case, which we will not discuss further, indicates the material would collapse onto a plane.

With the aid of the constitutive relations, Eqs. (3.24) and (3.25) can be transformed into the equations for the principal stresses and $\psi$, and Eqs. (3.4) and (3.11) can be transformed into the equations for the principal stretches. The five quantities $t_{1}, t_{2}, \psi, M(\alpha)$, and $D(\alpha)$ satisfy one of (3.24) or (3.25) and Eqs. (3.4) and (3.11); 
these were derived from the equations of equilibrium and the compatibility conditions. At the same time $\varphi$ may be found by integrating (3.15) or (3.17). Thus we can choose two of the five variables independently and then determine the others from the equations.

Before concluding this section, we note the following special cases.

(1) First suppose $\psi^{\prime}(\alpha)=0$, so that $\psi=$ constant ; then the principal axes do not change directions. For, without loss of generality, we may assume $\psi=0$. Then Eq. (3.4) requires $t_{2}^{\prime}=0$ but $t_{1}^{\prime} \neq 0$ (for inhomogeneous deformation) or $t_{1}^{\prime}=0$ but $t_{2}^{\prime} \neq 0$. Assume the former holds; then (3.11) yields

$$
M(\alpha)=0 .
$$

Therefore the lines are parallel, and further they are parallel to the $x_{1}$-axis. Introducing (3.26) into (3.24) we obtain

$$
g_{1}(\alpha)=C_{2} D^{\prime}(\alpha)
$$

with $g_{1}(\alpha)$ being given by

$$
g_{1}(\alpha)=-\lambda_{1} \lambda_{2} \frac{d}{d \alpha}\left(\frac{1}{2 \lambda_{1}^{2}}\right)=\frac{\lambda_{2} \lambda_{1}^{\prime}}{\lambda_{1}^{2}} .
$$

If we further specify $D^{\prime}(\alpha) \neq 0$ and the response relation $t_{2}=t_{2}\left(\lambda_{1}, \lambda_{2}\right)$, then $\lambda_{1}, \lambda_{2}$ may be determined from

$$
t_{2}\left(\lambda_{1}, \lambda_{2}\right)=t_{20}, \quad \frac{\lambda_{2} \lambda_{1}^{\prime}}{\lambda_{1}^{2}}=C_{2} D^{\prime}(\alpha),
$$

with $t_{20}$ being a constant.

(2) The second case is that $M(\alpha)=0$, i.e., $M^{\prime}(\alpha)=0$. Then Eqs. (3.11) and (3.24) yield

$$
\begin{gathered}
\left(t_{1}-t_{2}\right) \cos \psi \sin \psi=C_{4}, \\
\frac{d}{d \alpha}\left[\frac{\cos ^{2} \psi}{\lambda_{1}^{2}}+\frac{\sin ^{2} \psi}{\lambda_{2}^{2}}\right]=-\frac{2 C_{2} D^{\prime}}{\lambda_{1} \lambda_{2}} \frac{\alpha)}{}
\end{gathered}
$$

with $C_{4}$ being a constant. The principal stresses $t_{1}, t_{2}$, and the angle $\psi$ should be determined from (3.4), (3.29), and (3.30) when $D^{\prime}(\alpha)$ and the response relations are given.

For these first two cases where $M(\alpha)=0$, we have from (3.10) that $x_{2}=D(\alpha)$, so that $\alpha=\alpha\left(x_{2}\right)$; i.e., $\alpha$ does not depend on $x_{1}$.

(3) In the third case take $M(\alpha)=\tan \alpha$ and $D(\alpha) \equiv D_{0}=$ constant; then Eq. (3.10) gives

$$
\alpha=\arctan \left(\frac{x_{2}-D_{0}}{x_{1}}\right) .
$$

Thus the straight lines intersect at a point on the $x_{2}$-axis, and Eqs. (3.11) and (3.25) 
become

$$
\begin{gathered}
t_{1}^{\prime} \cos \psi \sin (\psi-\alpha)-t_{2}^{\prime} \sin \psi \cos (\psi-\alpha)+\left(t_{1}-t_{2}\right) \psi^{\prime} \cos (2 \psi-\alpha)=0, \\
\frac{\lambda_{1}^{\prime}}{\lambda_{1}^{3}} \cos ^{2}(\psi-\alpha)+\frac{\lambda_{2}^{\prime}}{\lambda_{2}^{3}} \sin ^{2}(\psi-\alpha)+\left(\frac{1}{\lambda_{1}^{2}}-\frac{1}{\lambda_{2}^{2}}\right) \psi^{\prime} \cos (\psi-\alpha) \sin (\psi-\alpha)=\frac{C_{2}}{\lambda_{1} \lambda_{2}} .
\end{gathered}
$$

The principal stresses and the angle may be determined from (3.4) and (3.31) when the response relations are given.

4. Example-straightening of a circular arc. In this section we will show, by example, that there is an inhomogeneous deformation in which the principal stresses (or strains) are constant and the Eulerian principal axes do not change direction along a straight line. The example considers the straightening of a circular arc. Introducing the cylindrical polar coordinate system $\left(R, \boldsymbol{\Theta}, X_{3}\right)$ in the undeformed configuration with

$$
R=\left(X_{1}^{2}+X_{2}^{2}\right)^{1 / 2}, \quad \boldsymbol{\theta}=\arctan \left(\frac{X_{2}}{X_{1}}\right),
$$

we can describe the deformation as

$$
\begin{aligned}
& x_{1}=f(\theta)=f\left(\arctan \left(\frac{X_{2}}{X_{1}}\right)\right), \\
& x_{2}=g(R)=g\left(\left(X_{1}^{1}+X_{2}^{2}\right)^{1 / 2}\right), \\
& x_{3}=\lambda X_{3} .
\end{aligned}
$$

Therefore the in-plane deformation gradient tensor and the left Cauchy-Green deformation tensor are

$$
\begin{gathered}
\mathbf{A}=\left(\begin{array}{cc}
-f^{\prime}(\boldsymbol{\theta}) \frac{X_{2}}{X_{1}^{2}+X_{2}^{2}} & f^{\prime}(\boldsymbol{\theta}) \frac{X_{1}}{X_{1}^{2}+X_{2}^{2}} \\
g^{\prime}(R) \frac{X_{1}}{\left(X_{1}^{2}+X_{2}^{2}\right)^{1 / 2}} & g^{\prime}(R) \frac{X_{2}}{\left(X_{1}^{2}+X_{2}^{2}\right)^{1 / 2}}
\end{array}\right), \\
\mathbf{B}=\mathbf{A} \mathbf{A}^{-1}=\left(\begin{array}{cc}
\frac{\left[f^{\prime}(\boldsymbol{\theta})\right]^{2}}{R^{2}} & 0 \\
0 & {\left[g^{\prime}(R)\right]^{2}}
\end{array}\right) .
\end{gathered}
$$

It follows that the Cauchy principal axes coincide with the coordinate axes; so $\psi=0$ everywhere, and the in-plane principal stretches are

$$
\lambda_{1}=\frac{f^{\prime}(\boldsymbol{\theta})}{R}=\frac{f^{\prime}\left(f^{-1}\left(x_{1}\right)\right)}{g^{-1}\left(x_{2}\right)}, \quad \lambda_{2}=g^{\prime}(R)=g^{\prime}\left(g^{-1}\left(x_{2}\right)\right) \text {. }
$$

Here we have assumed that the inverses of the functions $f(\boldsymbol{\theta})$ and $g(R)$ exist.

The stretch $\lambda_{2}$ is a function of $x_{2}$ alone; therefore the parameter function $\alpha$ must depend on $x_{2}$ only, i.e., $\alpha=\alpha\left(x_{2}\right)$, in order to express $\lambda_{2}$ in terms of $\alpha$. Furthermore $f^{\prime}(\theta)$ must be constant so that $\lambda_{1}$ can be expressed in terms of $\alpha$ too. Thus we have

$$
x_{1}=f(\boldsymbol{\theta})=C_{4} \boldsymbol{\theta}+x_{10}, \quad x_{2}=g(R)
$$


and

$$
\lambda_{1}=C_{4} / R=C_{4} / g^{-1}\left(x_{2}\right)=\lambda_{1}(\alpha), \quad \lambda_{2}=g^{\prime}\left(g^{-1}\left(x_{2}\right)\right)=\lambda_{2}(\alpha),
$$

with $C_{4}$ and $x_{10}$ being constants. The angle $\psi$ is zero everywhere, and along the straight lines $x_{2}=$ a constant,$\lambda_{1}$ and $\lambda_{2}$ do not change value. Thus the class of deformations (4.6), which is not homogeneous, satisfies the assumptions embodied in (3.1) and (3.2); and the straight lines $x_{2}=$ a constant are the special principal stress contours we are seeking. Note that this conclusion holds for any general isotropic material.

Since in this class of deformations $\psi \equiv 0$, we have the special case (1) discussed at the end of the last section. Therefore the principal stretches $\lambda_{1}$ and $\lambda_{2}$ are to be determined from (3.28) when the response relations are given. We have

$$
\lambda_{1}^{\prime} \equiv \frac{d \lambda_{1}}{d \alpha}=\frac{d \lambda_{1}}{d x_{2}} \frac{d x_{2}}{d \alpha}=D^{\prime}(\alpha) \frac{d \lambda_{1}}{d R} / \frac{d x_{2}}{d R}=-\frac{D^{\prime}(\alpha)}{C_{4}} \lambda_{1}^{2} / \lambda_{2} ;
$$

thus the second equation of (3.28) becomes $C_{2} C_{4}=-1$, which just relates two constants and determines the constant $C_{4}$ for the principal stretch $\lambda_{1}$. The principal stretch $\lambda_{2}$ or the function $g(R)$ may then be determined from the first equation of (3.28).

5. Conclusion. In this paper it has been shown that in nonlinear plane deformation the straight line is the only possible principal stress contour on which the principal stretches (stresses) are of constant value and the principal axes do not change direction. It has also been shown that the principal stress contours in the deformation for the straightening of a circular arc are such straight lines.

Acknowledgment. The work in this paper was initially carried out at the University of Nottingham and modified at the University of Queensland. The author is very grateful to Dr. W. A. Green for his suggestion of the problem and constructive guide on the initial research work. Thanks are also to Dr. V. G. Hart and Dr. J. A. Belward for their helpful discussion and careful check. The financial supports of the Agricultural Ministry of the People's Republic of China and the Department of Mathematics of the University of Queensland are acknowledged.

\section{REFERENCES}

[1] R. W. Ogden, Principal stress and strain trajectories in nonlinear elastostatics, Quart. Appl. Math. 44, 255-264 (1986)

[2] R. W. Ogden, Non-linear elastic deformations, Ellis Horwood, Chichester, 1984

[3] E. Varley and E. Cumberbatch, The finite deformation of an elastic material surrounding an elliptical hole, Finite Elasticity 27, ASME, 41-64 (1977)

[4] E. Varley and E. Cumberbatch, Finite deformations of elastic materials surrounding holes, J. Elasticity 10, 341-405 (1980)

[5] A. J. M. Spencer, Continuum Mechanics, Longman, London, 1980 